\title{
The Usefulness of "Analysis of Burden of Irritable Bowel Syndrome From National Health Insurance Claims Database" in the Healthcare Policy in Korea
}

\author{
Kyoung Sup Hong \\ Division of Gastroenterology, Department of Internal Medicine, Seoul National University College of Medicine and Seoul National University \\ Hospital, Seoul, Korea
}

Article: Estimating the burden of irritable bowel syndrome: analysis of a nationwide Korean database Jung HK, Kim YH, Park JY, et al.

(J Neurogastroenterol Motil 2014;20:242-252)

In Korea, all citizens are automatically registered for the compulsory insurance program, the National Health Insurance (NHI) system. The NHI allows citizens to access freely primary and secondary care without making an appointment in advance, which is one of the necessary steps in Western countries. NHI also provides easy access to tertiary care only with a referral form which could be issued at primary or secondary care clinic according to patient's request. The Health Insurance Review and Assessment (HIRA) is a service claims database of NHI which includes a nationwide, population-based health insurance electronic record of diagnostic and therapeutic claims in Korea.

When looking at costs for a disease, there are three different kinds of costs, namely direct, indirect and intangible costs. ${ }^{1}$ Direct costs are expenditures incurred by the healthcare system in managing the disease. Indirect costs are mainly related to production losses arising from the absence from work. Intangible costs are related to the pain and impaired quality of life (QOL) and are more difficult to measure.

The various economic elements of the burden of a given disease can be quantified in several ways. ${ }^{1}$ The most frequently adopted method is the prevalence approach, which typically examines a period of one year and, in case of direct healthcare costs. In those diseases where there exists an unambiguous definition of the condition and a unique identification number in the International Classification of Diseases (ICD), it is a straightforward process to draw on national and local data source, as well as extrapolations from studies containing any relevant resource utilization information, to construct a profile of the direct cost burden.

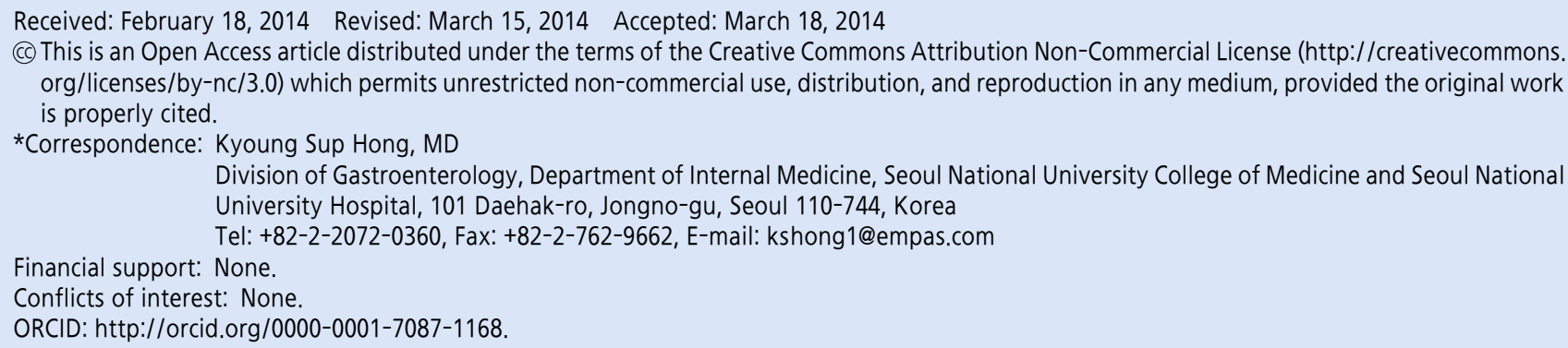


However, this would not be the case with irritable bowel syndrome (IBS). ${ }^{1}$ Although there is the International Statistical Classification of Diseases and Related Health Problems 10th Revision (ICD-10) codes which refer to IBS, it is uncertain how accurately the volume of resource utilization coded under this heading reflects the true magnitudes involved because of the diagnostic ambiguities surrounding IBS.

According to the fourth national morbidity study in general practice in the United Kingdom (UK) and a survey from consultant general gastroenterologists in the north England, ${ }^{1-2}$ the average number of consulting for IBS was 1.6 and 2 each year per patient, respectively. A small proportion, about $2.5 \%$, admitted to a hospital for an average stay of 5 days. Annual direct cost on IBS by the UK National Health Service (NHS) has been estimated to be 45.6 million pounds ( $£$ ), using 1995 data. This figure is equivalent to just $0.1 \%$ of total NHS annual spending and represents approximately $£ 90$ per patient. Using national database, Sandler et $\mathrm{al}^{3}$ reported that the yearly direct cost for IBS was calculated as $\$ 1.3$ billion, where hospital inpatient services accounted for the majority of these costs.

An alternative to the prevalence approach to illness costing is the incidence-based methodology which seeks to estimate the lifetime costs of managing a cohort of cases first diagnosed in a given year. ${ }^{1}$ However, this approach is much more demanding in terms of data and the information systems required. A cohort-based approach to measure the direct costs of IBS over a 1-year period has been employed by Talley et $\mathrm{al}^{4}$ in the US. This study used a billing database linked to all local sources of medical care and analyzed health service charges in relation to IBS status in a population of 3022 white people. This sample comprised 536 subjects (18\%) who had symptoms indicating IBS, 1711 (57\%) who had other gastrointestinal symptoms but did not meet the diagnostic criteria for IBS and 775 (25\%) who did not have any gastrointestinal symptoms. The IBS related excessive charge was $\$ 313$ per patient for the study year. Extrapolation of Talley's findings to the US white population suggests an annual excess charge due to IBS of $\$ 8$ billion, which is substantially different from the finding of national database by Sandler et al. ${ }^{3}$

Jung et $\mathrm{al}^{5}$ reported the epidemiologic features of IBS in 2008 and for the first time the medical and socioeconomic burdens it imposes on the Korean population through a cross-sectional study by using the NHI HIRA database which covers the entire population of Korea. The authors identified IBS and co-morbidities using the ICD-10 codes.

Among the Korean population who were at least 15 years of age, a total of 2,416,424 patients used medical care related to IBS during the 1-year period of 2008. The prevalence of IBS in this population was $6.9 \%$ in females and $5.1 \%$ in males, and tended to increase with age, ranging from $4.6 \%$ in $20-29$ year-olds to $8.7 \%$ in 60-69 year-olds. The proportion of IBS-D decreased with age with a prevalence of $48.1 \%$ in patients 20 years old or younger and $31.7 \%$ in older than 60 years, and was more prevalent in males $(38.3 \%)$ than in females $(33.8 \%)$. The mean visit time for outpatients was 2.5 per patient and $1.9 \%$ of the total IBS patients were admitted to a hospital bed for an average stay of 14.7 days. The healthcare system in Korea is a compromise between the UK and US systems, with the aims of accessibility and good coverage. Considering easier access to health care resources in Korea, the mean visit time and the proportion of admitted patients seem to be quite comparable to those of the UK.

The pharmaceutical usage and medical costs of 4,284,814 claims were analyzed. The total cost of IBS in 2008 was estimated to be $\$ 559$ million. Of this, the sum of direct medical cost, healthcare service and pharmacy cost, was $\$ 334$ million. The NHI costs for IBS, which include the NHI covered cost and beneficiary copayment charges, were estimated to be $\$ 155$ million, which corresponded to approximately $0.46 \%$ of the total direct cost for the entire Korean population during 2008. The annual average NHI direct cost for IBS was $\$ 64.1$ per person. Even considering the difference of annual income levels among Korea, the UK and the US, the direct cost per capita for IBS in Korea seems to be equal or less than those of the UK and the US.

For the first time in Korea, Jung et $\mathrm{al}^{5}$ conducted cross-sectional cost analyses using the NHI HIRA claims database, which covers the entire population of Korea. The authors presented considerable data concerning the burden of IBS in Korea, including prevalence, healthcare resource utilization, diagnostic and therapeutic cost, and indirect cost using the relevant socioeconomic data such as employment population ratio.

However, the diagnostic ambiguities surrounding IBS and the nature of a prevalence approach using the NHI claims database lead to 2 intrinsic limitations for accurate epidemiologic or cost analysis. First, there is no data to assure the diagnosis of IBS in the NHI claims database. Therefore, there is no way of gauzing how accurately IBS is being diagnosed and classified in the primary care setting and whether there is a tendency of over- or under-diagnosis of IBS. As a result, the epidemiologic features including prevalence might be biased. Second, with the lack of data about direct cost through personal spending and indirect cost, the estimation of total cost of IBS is difficult and is highly 
dependent on the methodology of each study. Therefore, the total cost estimates in the current study does not seem to be suitable for the comparison with those of other countries. Not only to overcome the limitations described above, the incidence-based prospective cohort study is needed also to estimate prevalence, direct and indirect cost of IBS in Korea more accurately.

Another downside of the current study is that there was no objective control to evaluate whether the direct cost for IBS in Korea is adequate or not. The authors stated that the direct medical cost of IBS was $30 \%$ of those related to cerebrovascular accidents, which was higher than those of depression or asthma in Korea. However, to assess the burden of IBS comprehensively, the intangible cost due to the impaired QOL should be considered as well as direct and indirect cost, which could be closely associated with QOL of IBS patients. In a Dutch study of IBS patients in different settings, hospital outpatients were found to report their complaints to be more serious and experience more daily life limitations than primary care patients. ${ }^{6}$ To establish the objective control from the patient's stand point, measurement of general QOL in IBS patients should be conducted before. Gralnek et $\mathrm{al}^{7}$ used SF-36 and showed that IBS patients seen in tertiary referral center demonstrated worse QOL than patients with gastroesophageal reflux disease, comparable QOL to patients with diabetes mellitus and end-stage renal disease, and better QOL than patients with depression. Therefore, the conditions showing comparable QOL impairment to IBS should be used as control to assess objectively the effectiveness of IBS cost in each country or ethnic group. A further development of cost-effectiveness analysis should be cost-utility analysis, which measures impact using quality-adjusted life years (QALYs). ${ }^{8}$ QALYs combine the length of life and QOL into a single number. To integrate outcome measurement in to the economic evaluation, preference-based measures, such as the EuroQOL 5D questionnaire can be used. ${ }^{9}$

IBS is notorious for both diagnostic ambiguity and no effective treatment so far, and is not considered as a good candidate for cost analysis to assess the disease burden in a population. Jung et $\mathrm{al}^{5}$ has just taken the first step to achieve greater efficiency in healthcare spending in Korea. However, at present, both gastroenterologists and healthcare decision makers in Korea should bear in mind the proverb 'Rome was not built in a day' and put their heads together to go further research into the economics of IBS.

\section{References}

1. Wells NE, Hahn BA, Whorwell PJ. Clinical economics review: irritable bowel syndrome. Aliment Pharmacol Ther 1997;11:1019-1030.

2. Charlton J, Skinner C. Fourth national morbidity study. Br J Gen Pract 1995;45:565.

3. Sandler RS, Everhart JE, Donowitz M, et al. The burden of selected digestive diseases in the United States. Gastroenterology 2002;122: 1500-1511.

4. Talley NJ, Gabriel SE, Harmsen WS, et al. Medical costs in community subjects with irritable bowel syndrome. Gastroenterology 1995;109:1736-1741.

5. Jung HK, Kim YH, Park JY, et al. Estimating the burden of irritable bowel syndrome: analysis of a nationwide Korean database. J Neurogastroenterol Motil 2014;20:242-252.

6. van der Horst HE, van Dulmen AM, Schellevis FG, van Eijk JT, Fennis JF, Bleijenberg G. Do patients with irritable bowel syndrome in primary care really differ from outpatients with irritable bowel syndrome? Gut 1997;41:669-674.

7. Gralnek IM, Hays RD, Kilbourne A, Naliboff B, Mayer EA. The impact of irritable bowel syndrome on health-related quality of life. Gastroenterology 2000;119:654-660.

8. Simrén M, Brazier J, Coremans G, et al. Quality of life and illness costs in irritable bowel syndrome. Digestion 2004;69:254-261.

9. Brooks R. EuroQol: the current state of play. Health Policy 1996; 37:53-72. 